\title{
L'espace sauvage: Merleau-Ponty et la pensée mécanique, picturale et poétique de l'espace
}

\author{
DANIEL OsKuI
}

Wie soll man den Raum behandeln, wenn er nichts als gefüllte Leere ist, in der sich Objekte befinden und sich Bewegung zwischen Objekten abspielt? Das klassische Denken des Raums, die Physik und Philosophie des 17. Jahrhunderts, bestimmen den Raum als einheitliche und ungeschiedene Umgebung, deren einzige Bedeutung darin besteht, als Bezugs-Rahmen für die geometrisch-mechanische Beschreibung des Raums zu dienen. Die folgende Arbeit versucht im Sinne der Phänomenologie seit Husserl einer originären Raum-Erfahrung nahe zu kommen, indem sie den phänomenologischen Beschreibungen und dem "fragenden Denken"von MerleauPonty folgt. Es gilt, diesseits des mechanischen Raums den "gelebten Raum" in seiner Vielgestaltigkeit und »Wildheit" wiederzufinden. In der geduldig phänomenologischen Beschreibung wird das Imaginäre aller Räumlichkeit hervortreten: auf der Grundlage einer originär vielgestaltigen Räumlichkeit erscheinen der poetische und der mechanische Raum schließlich als zwei komplementäre Raum-Erfahrungen.

\footnotetext{
Vertige! voici que frissonne

L'espace comme un grand baiser,

Qui, fout de naître pour personne,

Ne peut jaillir ni s'apaiser.

Mallarmé, Autre éventail
}

Les objets n'effleurent pas l'espace, ils se meuvent dans l'espace; l'espace accueille le spectacle d'objets, sans bouger lui-même; indépendants du spectateur, les objets ont une place définie: tels sont les postulats de la théorie classique de l'espace. Ainsi l'éventail de Mallarmé ne peut-il réellement toucher l'espace; il n'est qu'une image poétique, impuissant devant notre concept objectif de la spatialité.

L'espace classique semble correspondre à notre expérience quotidienne de l'espace: lorsque je ferme les yeux, lors d'un clin d'œil, les 
choses restent à leur place; quand je marche, mon corps se déplace dans l'espace comme les autres objets. L'espace nous accueille comme il accueille les objets. Il est le milieu unique et homogène. Séparé de toute matière, cet espace est une sorte de boîte vide à dimensions indéfinies. On a donc tendance à identifier l'espace vécu à l'espace physique et géométrique, à penser que nos actions se déroulent dans l'espace selon la dynamique classique.

Mais peut-on ramener à l'espace classique la variété et la richesse d'expériences spatiales? N'est-ce pas exclure les expériences enfantines, primitives, poétiques, oniriques, hallucinatoires de la spatialité? La conception classique ne nie pas l'existence de diverses formes de spatialité. Elle tend néanmoins à concevoir ces différentes formes à l'image de sa propre compréhension de la spatialité, à poser comme premier, comme fondement, comme norme sa notion d'espace. Ainsi Jean Piaget, lorsqu'il cherche à caractériser l'espace et la dynamique des objets perçus par l'enfant, s'appuie-t-il sur la mécanique et l'espace classiques. ${ }^{1} \mathrm{Il}$ est amené à ignorer, comme fait positif, l'expérience originaire de l'espace chez l'enfant, à la caractériser de manière toute négative, comme une expérience imparfaite et incomplète, en voie de développement vers la seule réalité et perfection géométrique de l'espace physique. De même, lorsqu'on cherche à caractériser la représentation spatiale de la peinture dite (primitives, on est tenté de la comparer avec la perspective centrale considérée comme la seule reproduction correcte de l'espace. N'est-ce pas considérer d'emblée comme dérivée, secondaire, adventice toute forme de spatialité autre que l'espace géométrico-physique?

On peut questionner l'exclusivité de l'espace classique en le resituant dans le temps: quelle est l'histoire de l'espace classique? Quel est son rapport à la genèse de la spatialité dans notre expérience? Peut-on réduire la dynamique de notre expérience de l'espace à la dynamique physique et à la spatialité qu'elle implique? On verra peutêtre apparaître des fissures dans cette construction homogène qu'est l'espace classique. Tel est l'objectif de ce travail. Je voudrais d'abord montrer en quel sens l'espace de la dynamique classique n'est pas une donnée, mais une acquisition historique, arrachée à d'autres conceptions possibles. Ensuite j'étudierai pourquoi ni l'explication objectiviste, ni l'explication rationaliste parviennent à rendre compte de la genèse de l'orientation spatiale. Finalement, j'essaierai de mettre en évidence, suivant le phénoménologue Merleau-Ponty, la genèse d'une

1. Jean Piaget, Bärbel Inhelder: La représentation de l'espace chez l'enfant, Paris 1948. Pour une discussion critique, cf. René Thom: La genèse de l'espace représentatif selon Piaget, in: L. Lurçat: Espace vécu et espace connu àl'école maternelle, Paris 1982, 164-170. 
spatialité originaire, émergeant de la communication sensible et pratique entre l'homme et le monde.

I.

\title{
L'espace physique et la perception spatiale mécanisée: brève histoire d'une conquête de l'imagination
}

\author{
Le haut n'est pas un lieu quelconque; \\ $c^{\prime}$ est le lieu où se dirige le feu \\ Aristote \\ Le repos est un mouvement rendu nul \\ parce que partagé \\ Galileo Galilei
}

Il n'y a pas de portrait simple de l'histoire de l'espace physique. C'est l'histoire d'une conquête plutôt que d'une découverte. Pour s'en rendre compte, il suffit de se pencher sur l'histoire de la dynamique, de la théorie du mouvement. Selon un portrait répandu, Galilée et Newton seraient parvenus au XVII ${ }^{\mathrm{e}}$ siècle, partant de l'observation systématique, à remplacer la dynamique aristotélicienne, alambiquée et incohérente, par une dynamique d'une simplicité, unité et cohérence stupéfiantes: si Aristote distinguait encore, dans l'univers, deux espaces différents («terrestre et supralunaire») où règnent des lois différentes, les Principia $^{2}$ de Newton, somme de la nouvelle dynamique, donne une description unifiée de l'espace. La pomme tombant d'un arbre et les planètes tournant autour du soleil sont désormais soumises aux mêmes lois universelles. Cette mécanique classique aurait trouvé la véritable définition du mouvement et de l'espace.

Mais ce portrait est simpliste. Sans pouvoir suivre ici, à l'instar de Paul Feyerabend, ${ }^{3}$ les méandres de l'histoire de la dynamique, notons-en un aspect décisif: l'élégante simplicité de la nouvelle dynamique ne provient pas de la nouvelle méthode expérimentale qui aurait remplacé le dogmatisme aristotélicien. Le sens épistémologique de la

2. Isaac Newton: De philosophiae naturalis principia mathematica, 1687 (trad. fr. par Christian Bourgois: Les principes mathématiques de la philosophie naturelle, Paris 1985).

3. Cf. Paul Feyerabend: Against Method. Outline of an Anarchistic Theory of Knowledge, London 1975 (trad. fr.: Contre la méthode, Paris 1979). Feyerabend cite dans son introduction Lénine: «l'histoire est toujours plus riche, plus variée, plus multiforme, plus vivante [...] que ne pensent les meilleurs partis, les avant-gardes les plus conscientes» (Lénin: La maladie infantile du communisme, Paris 1953, 87f.). 
méthode expérimentale et des nouvelles notions de mouvement et d'espace ne relève pas de l'empirie, mais de l'imagination scientifique, technique et rhétorique. Présenter la dynamique d'Aristote comme dogmatique du fait qu'elle a été soutenue par le Saint-Siège, c'est oublier que toute sa philosophie, notamment sa Physique plaide pour l'expérience, contre les concepts abstraits et géométrisants des pythagoriciens et de Platon. Est réel, pour Aristote, ce qui se montre de manière directe dans notre expérience. Ainsi la terre est-elle immobile pour au moins deux observations quotidiennes: nous ne pouvons percevoir aucun mouvement global de la terre et, une pierre qu'on lance d'un endroit verticalement vers le haut retombera toujours exactement à cet endroit. ${ }^{4}$ Présenter Galilée comme empiriste, parce qu'il aurait conçu les plans inclinés et les lunettes télescopiques, c'est méconnaître l'importance de la méthode mathématique pour sa science. Ni l'induction ni la déduction, chacune prise isolément, pouvaient amener le nouveau «système du monde». ${ }^{5}$ L'idée d'un espace uniforme naît d'un mariage entre induction (variation expérimentale élaborée) et déduction (description mathématisée), mariage très particulier et subtilement arrangé par Galilée. L'expérience de pensée, accomplie dans l'imagination, en est le procédé paradigmatique. ${ }^{6}$ Dès lors, la notion classique de l'espace est bien plus le résultat d'une conquête intellectuelle que d'une pure induction. Cette notion doit, même dans le seul domaine de la physique, s'imposer aux autres notions d'espace qu'elle combat.

4. Cette observation s'appellera plus tard l'argument de la tour: une pierre lâchée du haut d'une tour tombe toujours au pied de la tour; si la terre se déplaçait réellement dans l'espace, la pierre atterrirait à quelque distance du pied de la tour emportée entre-temps par la terre. Cf. Feyerabend: Against Method, op.cit., chap. 7; Pierre Duhem: Le système du monde. Histoire des doctrines cosmologiques de Platon à Copernic, Paris 1958 , t. I, 227-228.

5. Cf. Dialogue concernant les deux plus grands systèmes du Monde, où Galilée confronte avec une rhétorique élaborée l'héliocentrisme au géocentrisme. Je citerai d'après l'édition italienne, Galileo Galilei: Dialogo dei Massimi Sistemi del Mondo, in: Opera, Firenze 1964, t. III.

6. Cf. Feyerabend: Against Method, op.cit., chap. 7. Les «astuces» de Galilée «obscurcissent [...] le fait que l'expérience sur laquelle Galilée veut fonder les conceptions coperniciennes n'est rien d'autre que le résultat de sa propre imagination fertile» (ibid., 85). La visée de l'argumentation de Feyerabend n'est pas, bien entendu, de critiquer Galilée, mais de décrire la pratique intellectuelle et scientifique de Galilée dans toute sa richesse et ingéniosité, sans la ramener d'emblée à une épistémologie rationaliste réductrice. 


\section{L'interprétation naturelle de L'Espace chez Aristote et SA RÉiNTERPRÉTATION GÉOMÉTRIQUE ET RELATIVISTE PAR GALILÉE}

L'héliocentrisme de Copernic semble se heurter aux deux observations d'Aristote. Galilée, prenant la défense de Copernic, ne peut le nier: nous ne voyons pas bouger la terre et la pierre chute bien verticalement. Galilée en imagine pourtant une autre explication: ce qui chez Aristote contredit Copernic, ce ne sont pas les faits, mais l' «interprétation naturelle» de ces faits. ${ }^{7}$ L'interprétation naturelle tacite consiste à supposer l'identité entre le mouvement perçu et le mouvement réel, entre l'espace perçu et l'espace réel: ne bouge que ce que nous voyons bouger. Cette interprétation naturelle une fois identifiée, comment la contrer? Comment montrer que la Terre tourne, sans se faire remarquer, à une vitesse d'environ $100.000 \mathrm{~km} / \mathrm{h}$ comme on le sait aujourd' hui, autour du soleil? Pour défendre Copernic, Galilée n'a pas besoin de nier l'expérience quotidienne. Il suffit d'en proposer une autre interprétation, quitte à transformer radicalement le système notionnel de la dynamique (espace, mouvement, repos). Dans le Dialogo, Galilée laisse Salviati, personnage incarnant Copernic, présenter cette nouvelle interprétation dans différentes expériences de pensée, notamment celle-ci: des papillons, enfermés dans la cabine d'un navire, continueront à voler indifféremment dans toutes les directions de la cabine, pourvu que le mouvement soit uniforme et ne fluctue pas. «On ne les verra jamais s'épuiser à suivre le navire dans sa course rapide». ${ }^{8}$ On peut dès lors, tout en acquiesçant aux observations d'Aristote, réfuter son géocentrisme: De même que la pierre d'Aristote tombe toujours verticalement sur terre, une pierre chutant du mât du navire galiléen tombera au pied du mât. De même que, installé sur un navire traversant l'océan, on ne ressent pas nécessairement le mouvement du navire, on ne perçoit pas, vivant sur terre, sa course autour du soleil. L'espace perçu depuis la terre (idem depuis le navire) n'est pas l'espace réel.

Cette réinterprétation de notre expérience naturelle va de pair avec une redéfinition de l'espace physique. Pour Aristote, le mouvement d'un corps se comprend à partir d'un repère spatial absolu: chaque corps tend vers le «lieu qui lui est propre»; le lieu propre à la pierre est le centre de la terre, vers lequel elle tendra toujours dans sa chute,

7. Cf. Feyerabend: Against Method, op.cit., chap. 7.

8. Cf. Galilei: Dialogo, op.cit., 458. En vérité Galilée amène sur le navire une foule d'animaux et d'objets, indice d'une rhétorique foisonnante (Feyerabend: Against Method, op.cit., 85, parle de «propagande») qui lui a valu sa condamnation par le Saint Siège. 
pourvu que «rien ne s'y oppose». ${ }^{9}$ Ce mouvement naturel inscrit dans l'espace des directions déterminées: ‘vers le bas〉, ‘vers le haut〉, dessinant la dimension verticale. L'espace se polarise. Il n'est pas isotrope, il est orienté de façon absolue. La polarisation spatiale «ne montre pas seulement que le lieu est quelque chose de réel, mais encore qu'il a une certaine puissance». ${ }^{10}$ Comment attribuer une puissance de ce genre à l'espace géométrique? Contrairement aux pythagoriciens et à Platon, Aristote refuse d'assimiler l'espace physique à l'espace géométrique: le lieu ou la «situation` d'un objet ne se laisse pas réduire à sa position dans l'espace géométrique. ${ }^{11}$ Pour la même raison, Aristote refuse l'idée d'un espace vide: le mouvement n'est possible que dans un milieu orienté par une diversité de repères rendant possible la perception déterminée du mouvement. L'espace vide ou la chóra platonicienne, parfaitement homogène, n'offrent aucun repère: ${ }^{12}$ "dans le vide, il serait nécessaire que tout corps demeurât en repos; [...] car le vide ne présente aucune différence». ${ }^{13}$

$C^{\prime}$ est pourtant en définissant l'espace comme isotrope et vide que la mécanique du XVII ${ }^{\mathrm{e}}$ siècle fait un progrès prodigieux. Galilée et Newton se montrent platoniciens et géomètres. Une fois de plus, c'est l'expérimentation portée par l'imagination qui permet de démontrer l'idée mécanique du vide, selon la «méthode des variations proportionnelles` et du 〈passage à la limite: on constate d'abord qu'une boule lancée sur une surface poursuit d'autant plus longtemps son mouvement que la surface est lisse; on en conclut ensuite que sur une patinoire idéale, mieux encore dans l'espace vidé de tout obstacle (l'air, la gravitation, le dioxyde de carbone), la boule poursuivra indéfiniment son mouvement rectiligne et uniforme. L'espace vide identifié comme milieu idéal du mouvement, pourquoi favoriser tel ou tel système de coordonnées? L'‘expérience> des papillons n'illustre-t-elle pas la relativité du mouvement? En effet, peu importe si le navire est au port ou en pleine mer, le vol des papillons reste léger. Le mouvement n'existe que par rapport à un corps considéré en repos: le référentiel. Le repos n'est

9. Aristote: Physique d'Aristote ou Leçons sur les principes généraux de la nature, Paris 1862, tome II, livre IV, chap. II, 141 (trad. et annoté par Jules Barthélemy-Saint-Hilaire).

10. Aristote: Physique, livre IV, chap. II, op.cit., $140 f$.

11. L'erreur des platoniciens et des pythagoriciens est, aux yeux d'Aristote, semblable à celle d'un géomètre qui croirait qu'un cube possède en soi un côté droite et un côté gauche, indépendamment de sa situation par rapport à nous. Cf. Duhem: Le système du monde, op.cit., 188.

12. Cf. pour une analyse approfondie ibd. $189 \mathrm{ff}$.

13. Sur la critique de l'espace vide, cf. Aristote: Physique, op.cit., livre IV, chap. VIII. 
qu'un mouvement partagé par deux corps: «un mouvement rendu nul parce que partagé», selon la formule laconique de Galilée. ${ }^{14} \mathrm{Si}$ donc pour Aristote, l'espace est orienté de façon absolue, pour Galilée, l'espace n'est que le milieu parfaitement homogène d'infinis référentiels quelconques.

\section{Le mOdèle CARTÉSien de LA PERCEPTiOn SPATIALE: LA MÉCANIQUE GÉNÉRALISÉE}

Le divorce prononcé par la dynamique classique entre l'espace naturel absolu, et l'espace mécanique relatif, qui a inquiété Newton et inspiré Einstein, ${ }^{15}$ en implique un autre, déjà mentionné: le divorce entre l'espace perçu et l'espace réel. L'espace mécanique est-il plus réel, plus vrai que notre expérience de l'espace? En appliquant la mécanique à notre perception, Descartes, contemporain de Galilée, répond par l'affirmative.

Si la nouvelle mécanique délaisse la (méta)physique d'Aristote, que devient la philosophie? Descartes sera la figure emblématique à l'origine d'une nouvelle pensée. Pour sauver la métaphysique et la théologie, il inverse le sens de l'évolution scientifique: exerçant un

14. Galilée: Dialogo, op.cit., 378. Le conflit entre géocentrisme et héliocentrisme tourne en fin de compte autour de la relativité du mouvement: que l'observateur prenne le soleil ou la terre comme référentiel, dans les deux cas, il est possible de décrire mathématiquement la trajectoire des planètes. Il est vrai que la description de Copernic s'avère plus limpide. Mais cela n'est pas en soi une preuve de l'adéquation à la réalité céleste. Osiander, l'éditeur de Copernic, a justement su atténuer considérablement la virulence théologique de l'héliocentrisme en soulignant la différence nette entre hypothèse mathématique et existence réelle. Dans son «avis au lecteur», l'espace héliocentrique se présente comme un simple modèle permettant d'améliorer les calculs, «fiction calculatoire» un peu plus efficace que la réalité ptoléméenne.

15. Newton, esprit profondément religieux, ne pouvait accepter l'idée de l'univers vide - pourtant efficace à ses yeux comme principe d'explication physique. Ses sentiments théologiques étaient avec Descartes qui rejetait l'idée du vide, son intellect physique avec Galilée. Il conçoit l'idée d'un espace absolu invisible englobant tous les espaces relatifs sensibles (Principia, 9). À la fin du XIX siècle, Ernst Mach relance le débat, tout en condamnant l'espace absolu de Newton comme une idée «métaphysique». Il émet l'hypothèse que l'ensemble des corps célestes éloignés (les «étoiles fixes») définit le cadre de référence absolu. Einstein rejette finalement les essais de Newton et de Mach de déterminer un repère spatial absolu et généralise la relativité du mouvement: il jugeait peu esthétique, c'est-à-dire dérangeant la simplicité et la symétrie de la théorie dynamique, l'idée selon laquelle il existe différents types de référentiel, les lois de la mécanique n'y ayant pas la même forme. Cf. Albert Einstein, Léopold Infeld: L'évolution des idées en physique, Paris 1983, 197-202. 
doute radical pour faire table rase de l'ancienne philosophie, il cherche à rétablir la pensée philosophique en y introduisant et généralisant, paradoxalement, ces mêmes concepts mécaniques qui sont en train d'abolir l'ancienne philosophie. ${ }^{16}$ Cela devient décisif pour la question de l'espace: après avoir radicalement séparé le cogito (la «substance pensante») de la nature matérielle (la «substance étendue»), Descartes généralise la physique mécanique pour l'appliquer à toute la nature, surtout à l'homme lui-même. «La nature de la matière ou du corps [...] ne consiste point en ce qu'il est une chose dure, ou pesante, ou colorée [...], mais seulement en ce qu'il est une substance étendue en longueur, largeur, et profondeur». ${ }^{17}$ Voilà l'essence de la res extensa, étonnamment abstraite, voire vide: la nature est l'espace mécanique. Comme Galilée, Descartes sépare le réel du perçu, la qualité première de la qualité secondaire, la physique de notre expérience, tout en exacerbant ces distinctions. ${ }^{18} \mathrm{Il}$ géométrise la nouvelle physique pour l'ériger en métaphysique. Désormais toute la nature, animée aussi bien qu'inanimée, n'est définie que par l'étendue, la divisibilité et le mouvement mécanique. L'homme biologique, de même que l'animal, ne peut être non seulement expliqué comme une machine: il est une machine.

Dans La Dioptrique de 1637, où Descartes applique ses principes méthodiques et métaphysiques à la perception visuelle, la dépréciation de l'expérience s'annonce dès la première page: Descartes déplore que l'invention des «merveilleuses lunettes» télescopiques fut laissée au hasard, le hollandais Jacob Metuis ne les ayant découvertes «que par expérience et fortune», et non pas par déduction scientifique. ${ }^{19} \mathrm{La}$

16. Aux yeux de Descartes, l'évolution scientifique de son temps provoque une mêlée absurde: la théorie mécanique (vraie pour Descartes) critiquant la scolastique (fausse) risque d'atteindre la religion (vraie) et, inversement, la religion, en couvrant la scolastique, risque d'entraver le mécanisme. On risque donc de perdre sur les deux tableaux. Le doute radical, le désir de faire table rase provient de là.

17. René Descartes: Les principes de la philosophie, CEuvres de Descartes, Vol. 3, publ. par Victor Cousin, Paris 1995, $2^{\mathrm{e}}$ partie, principe 4, 123.

18. Si Galilée apparie induction et déduction (dans l'imagination expérimentale), Descartes favorise la déduction mathématique au détriment de notre expérience sensible. Descartes semble avoir formulé l'antique distinction atomiste, entre qualité première et qualité secondaire, impliquée dans la définition de la res extensa, indépendamment d'Il saggiatore de Galilée, cf. Harald Höfding: Histoire de la philosophie moderne, t. I, Paris 1906, 132-133.

19. «Mais, à la honte de nos sciences, cette invention, si utile et si admirable, n'a premièrement été trouvée que par l'expérience et la fortune», Descartes: La dioptrique, discours $\mathrm{I}^{\mathrm{er}}, 3-4$. Entre 1570 et 1610 , différentes personnes revendiquèrent l'invention des «lunettes télescopiques», qui devront profondément transformer l'espace 
Dioptrique se propose précisément de combler cette lacune à la fois métaphysique et technique. ${ }^{20}$ Comment définit-elle l'espace visuel? Plusieurs analogies illustrent les explications. La plus connue et la plus surprenante est celle qui compare la vision à un aveugle se servant de son bâton comme d'un «sixième sens». Ainsi dans la définition de la lumière comme phénomène mécanique:

«la lumière n'est autre chose, dans les corps qu'on nomme lumineux, qu'un certain mouvement, ou une action fort prompte et fort vive, qui passe vers nos yeux [...], en même façon que le mouvement ou la résistance des corps, que rencontre cet aveugle, passe vers sa main, par de son bâton.» ${ }^{21}$

La vision serait-elle un aveugle? La mécanique de la perception ne pourrait se passer d'une telle métaphore - de la métaphore? Quoiqu'il en soi, cette comparaison ne nous quittera plus. Toutes les définitions de $L$ a dioptrique seront explicitées par elle. Ainsi la définition de la couleur: de même que l'aveugle peut distinguer «des arbres, des pierres, de l'eau, et choses semblables, par l'entremise de son bâton [...]», de même nous percevons «le rouge, le jaune, le vert, et toutes les autres couleurs» en fonction de la «façon» dont les choses reflètent les mouvements lumineux. C'est donc en fonction des lois de la projection géométrique, définies dans l'espace euclidien que les qualités premières de la vision seront expliquées. On entrevoit ici comment la géométrie analytique - inventée par Descartes lui-même - s'allie à la mécanique du mouvement lumineux, le bâton de l'aveugle illustrant les lignes de projection. Quant à l'espace perceptif, il n'enferme plus aucun secret. Il est circonscrit par six qualités premières: «la lumière, la couleur, la situation, la distance, la grandeur, et la figure», toutes définies more geometrico. $^{22}$

visuel et notre vision d'espace: par exemple William Bourne pour son père Thomas Digges, Hans Lippershey, qui en déposa le premier brevet, refusé, en 1608.

20. Cet incipit exposant la portée technique de La dioptrique sur le fond de la nouvelle métaphysique, contient un nombre étonnant de contradictions subtiles: si la valeur de l'expérience est secondaire, pourquoi vouloir inventer, par déduction, un instrument, «si utile et si admirable» qui ne peut servir qu'à l'élargissement de notre champ d'expérience?

21. Descartes: La dioptrique, op.cit., discours $I^{\mathrm{er}}, 6 \mathrm{f}$.

22. Ibid., discours $V I^{e}, 54$. Bien entendu, ces qualités ne sont pas les qualités - secondaires - telles que nous les percevons, mais les «qualités principales» de la vision, telles qu'elles sont définies dans la mécanique optique. Ainsi la luminosité est-elle définie par la «force des mouvements» qui agissent sur la rétine, la couleur par la «façon de ces mouvements» (ibid., 55). Je reviendrai sur la définition cartésienne de «situation» et de «distance» plus en détail du point de vue critique de la phénoménologie. 
Ainsi le rapport entre expérience et espace s'inverse-t-il: la notion physique d'espace n'apparaît plus comme extraite de notre perception spatiale par l'abstraction imaginative que Galilée pratique; c'est notre perception elle-même que Descartes soumet à la mécanique spatiale. L'expérience de l'espace n'explique rien, c'est elle qu'il s'agit d'expliquer. Tel est le sens épistémologique et ontologique de la notion de qualité secondaire. La physique prenant ainsi la place de la métaphysique, la question philosophique de l'expérience ne peut plus être celle que pose Aristote: quel est cet espace, ce milieu où nous percevons les choses? ${ }^{23}$ Il ne s'agit plus de décrire l'expérience spatiale comme elle nous apparaît, dans toute sa richesse, pour ensuite seulement en extraire des traits essentiels. L'interrogateur cartésien doit se restreindre d'emblée: comment percevons-nous les choses dans l'espace physique? Quels en sont les mécanismes? Ainsi la vision peut-elle être comparée à l'aveugle, parce qu'elle a été réduite à la mécanique. ${ }^{24}$ Paradoxalement, en adoptant le modèle mécanique, qui est un modèle du toucher et du contact, Descartes doit renoncer à rester au contact de son expérience visuelle, dénigrée comme qualité secondaire. Il n'a plus le droit de la décrire, de l' «interroger», ${ }^{25}$ telle qu'elle lui apparaît: malgré le modèle du contact, constate le phénoménologue Merleau-Ponty, «nul souci de coller à la vision». ${ }^{26}$

23. Cette question complexe et centrale de la quiddité de l'espace préoccupe Aristote dans la Physique pendant longtemps: chapitres I à VII du livre IV. En ce sens, la théorie dynamique d'Aristote était une théorie du changement en général, comprenant non seulement la locomotion, mais également le changement qualitatif, la génération et la corruption; elle fournissait même, comme fait remarquer Feyerabend, «une base à la théorie de la sorcellerie» (Feyerabend: Against Method, op.cit., 108).

24. Comme mécanisme perceptif, la vision et le toucher ne se distinguent plus, toute différence entre eux relevant des qualités secondaires. Qui plus est, la vision doit être ramenée au toucher; car le modèle mécanique lui-même provient de l'expérience du contact tactile.

25. C'est la critique phénoménologique que notamment Merleau-Ponty adresse à la pensée cartésienne, de ne plus être vrai questionnement philosophique, «pensée interrogative».

26. Maurice Merleau-Ponty: L'œil et l'esprit, Paris 1964, 36. 


\section{II. \\ La critique phénoménologique: la genèse de l'espace entre l'espace objectif et l'espace pensé}

Notre vision de l'espace «se laisse» réduire à l'espace géométrico-physique. ${ }^{27}$ Voilà l'enseignement de la mécanique généralisée du XVII siècle. L'homme peut appliquer sa théorie mécanique de la nature à lui-même; il peut chercher à expliquer sa propre expérience selon le modèle qu'il a construit à partir d'elle. ${ }^{28}$ Mais à quel dessin s'objectiver soi-même de cette manière? Est-ce le désir de se mirer?

Il était nécessaire et bénéfique, pour construire un modèle mécanique unifié, pour rendre la nature chiffrable, d'abandonner la physique qualitative d'Aristote et l'expérience quotidienne où elle était enracinée. Depuis, la science galiléenne a inauguré, on le sait, l'histoire de la technique et la technique a transformé notre expérience. ${ }^{29}$ L'ordre des chapitres dans La dioptrique cartésienne en est déjà une image: elle définit d'abord «la lumière», ensuite «la vision», pour enfin traiter scientifiquement «des moyens de perfectionner la vision» et «de la façon de tailler les verres». ${ }^{30}$

Toutefois, chaque progrès projette ses ombres. Donnant naissance aux multiples disciplines scientifiques qui s'émancipent de la philosophie, y réclamant un monopole méthodologique, la science galiléenne a provoqué divers mouvements d'opposition intellectuelle et sociale. Ces mouvements mettent en évidence une «crise» dont la phénoménologie tardive de Husserl a formulé, de manière représentative,

27. "Toutes les qualités que nous apercevons, peuvent être réduites à six principales: la lumière, la couleur, la situation, [...]», Descartes: La dioptrique, op.cit., 54. «Peut être réduit», c'est une formule récurrente dans La dioptrique, significative du programme cartésien.

28. C'est le projet même des «sciences cognitives» de l'esprit.

29. Qu'importe Aristote, du point de vue de l'expérience naturelle qui n'est pas encore technique de la nature, la quantification? Qu'importe de vérifier méticuleusement, à la manière de Galilée, si la pomme lourde tombe réellement plus rapidement que la pomme légère? L'explication qualitative que donne Aristote répond à son expérience de la réalité. C'est là l'essence de sa métaphysique de la 〈physis〉, englobant la nature, l'homme, l'animal, la plante et la pierre, tous animés d'une tendance (la pierre), d'une âme végétative (la plante), sensitive (l'animal) ou intellective (l'homme). La pomme, chutant, aspire à retrouver son lieu d'origine - comme l'oiseau migrateur.

30. Le XVII ${ }^{\mathrm{e}}$ siècle est en même temps le siècle où on libère la pensée rationnelle de l'emprise du pouvoir ecclésiastique. C'est dans ce triple sens, scientifique, technique et politique, que nous avons parlé d'une »conquête« de l'espace mécanique. 
les traits essentiels: ${ }^{31}$ la science moderne s'est détournée de sa véritable vocation, que depuis la Grèce les grands penseurs ont rêvé comme étant la prise de conscience par l'homme de sa destination rationnelle et spirituelle. Si les sciences de la nature ont connu un succès indéniable, elles ont échoué en appliquant leur méthode si féconde au domaine de l'esprit, à l'homme lui-même. L'échec est dû à deux formes d'erreur et d'oubli: la réduction des différentes formes de rationalité à la seule raison technique; l'éloignement, voire la séparation de notre expérience, de notre rapport naturel, sensible et intégral au monde et à l'Être. Ainsi le phénoménologue Merleau-Ponty, dans son dernier essai L'œil et l'esprit commence-t-il son interrogation de l'«être-au-monde» par le constat d'une aliénation:

«La science manipule les choses et renonce à les habiter. Elle s'en donne des modèles internes et, opérant sur ces indices ou variables les transformations permises par leur définition, ne se confronte que de loin en loin avec le monde actuel». ${ }^{32}$

Descartes est devenu l'emblème de cette aliénation. C'est à son nom qu'on associe la scission entre la nature et l'esprit, entre le corps et l'âme, entre pensée et expérience sensible. La compréhension de l'espace se restreint ainsi à l'alternative entre l'empirisme (l'objectivisme) qui réduit l'espace aux objets juxtaposés dans l'espace («espace spatialisé») et l'intellectualisme (le rationalisme) qui construit l'espace comme synthèse du sujet indépendamment de la matière de notre expérience («espace spatialisant»). ${ }^{33}$ «Se confronter avec le monde actuel»: toute la pensée phénoménologique de Merleau-Ponty est marquée par ce désir de retrouver et de décrire à nouveau notre lien primordial au monde de l'expérience sous ses formes multiples. L'homme est un «être-au-monde», il n'est pas simplement «dans» le monde. Il s'agit de retrouver notre expérience de l'espace, là où elle surgit, dans la dynamique de notre expérience, avant son figement par l'empirisme et l'intellectualisme.

\section{LA QUESTION DE LA DIMENSIONNALITÉ: L'EXEMPLE DE LA VERTICALITÉ}

Contre la relativité de l'espace physique, abstraction efficace opérée par la mécanique classique et einsteinienne, la phénoménologie s'attache à un ‘fait` simple de notre expérience quotidienne: l'espace

31. Cf. par exemple Edmund Husserl: La crise de l'humanité européenne et la philosophie, Paris 1992.

32. Merleau-Ponty: L'œil et l'esprit, op.cit., 9.

33. Merleau-Ponty: Phénoménologie de la perception, Paris 1945, 282, 287. 
nous apparaît comme étant orienté, selon un ici absolu. D'où jaillit l'orientation spatiale? C'est cette question généalogique que MerleauPonty aborde dans un important chapitre de la Phénoménologie de la perception, consacré à l'espace. Il s'agit de revenir sur le pas du XVII siècle mécaniste, pour reprendre d'une manière phénoménologique l'interrogation aristotélicienne et lui donner une autre direction. «Renversement de la révolution copernicienne»: c'est la devise de Husserl dans un inédit que Merleau-Ponty a pu consulté ${ }^{34}$ Le sol originaire de l'expérience, contrairement à la terre-planète ne bouge pas. ${ }^{35} \mathrm{Com}$ ment comprendre la possibilité d'un tel repère absolu?

«Soit, par exemple, notre expérience du «haut〉 et du 〈bas»»: $:^{36}$ Merleau-Ponty, comme Aristote, commence par étudier la dimension de la verticalité. Ce choix n'est pas anodin. Pour Aristote, nous l'avons $\mathrm{vu}$, la verticalité est la dimension exemplaire du caractère objectif de l'orientation spatiale: «le haut n'est pas un lieu quelconque; c'est le lieu où se dirige le feu, et en général les corps légers». ${ }^{37}$ Pourtant, si Aristote n'a pas de difficulté à expliquer le mouvement naturel des éléments vers le bas (la terre et l'eau) et vers le haut (le feu et l'air), il ne parle pas explicitement du mouvement selon les deux autres dimensions, horizontale et transversale, et il ne peut en donner aucun exemple. ${ }^{38}$ Serait-ce donc un phénomène physique, la gravitation, qui expliquerait l'orientation objective selon la seule verticalité? Faudrait-il distinguer, à la manière cartésienne, la verticalité comme «qualité objective» des deux autres dimensions dépréciées comme «qualités subjectives»? La tâche de Merleau-Ponty sera précisément de montrer que l'alternative entre aristotélisme et cartésianisme est asymétrique et insatisfaisante. Si Merleau-Ponty commence par la question de la verticalité, ce n'est pas pour la privilégier au détriment de deux autres dimensions, mais bien plus pour poser une question plus large : la question de la «dimensionnalité» en générale, en deçà de l'empirisme physique et de l'intellectualisme géométrique. L'étude de la verticalité est donc en ceci exemplaire qu'elle exacerbe les difficultés de cette alternative.

34. Cf. Edmund Husserl: La terre ne se meut pas, Paris 1989.

35. «Je ne suis pas en déplacement; que je me tienne tranquille ou que je marche, ma chair est le centre et les corps en repos et mobiles sont tout autour de moi et j'ai un sol sans mobilité», Husserl: La terre, op.cit., 17.

36. Merleau-Ponty: Phénoménologie, op.cit., 282.

37. Aristote: Physique, op.cit., livre IV, chap. II, 141.

38. Il n'en est question nulle part dans les chapitres I à VII de la Physique, concernant l'espace et le mouvement. 
2. LA GENÈSE de LA VERTICALITÉ: UNE EXPÉRIENCE GESTALTISTE ET POURQUOI NI L'EMPIRISME NI L'INTELLECTUALISME NE PEUVENT L'EXPLIQUER?

Comment saisir notre expérience originaire du 〈haut〉 et du 〈bas» «avant toute élaboration notionnelle», si les sciences et leurs notions ont transformé cette expérience, si elle n'est plus vraiment «notre` expérience? L'expérience originaire n'est donc pas l'expérience ordinaire de la vie, «car elle est alors dissimulée sous ses propres acquisitions», notamment sous la conception mécanique de l'espace. ${ }^{39}$ Dans cette situation, Merleau-Ponty nous propose un détour par la psychologie de la perception, par l'expérience scientifique qui provoque «quelque cas exceptionnel». ${ }^{40}$ Il réaffirme ainsi son ferme espoir que les sciences galiléennes de l'homme ne soient pas condamnées à réduire l'homme à une chose, mais puissent faire entrevoir son être. ${ }^{41}$ Merleau-Ponty se tourne notamment vers la psychologie de la Gestalt (forme) à l'aide de laquelle il cherche à dépasser les notions dichotomiques et abstraites issues du cartésianisme.

Àux quels «cas exceptionnels» Merleau-Ponty pense-t-il? C'est d'abord «la vision sans inversion de l'image rétinienne», une fameuse série d'expériences présentées par le gestaltiste G.M. Stratton à partir de $1896 .^{42}$ Ce scénario expérimental permet d'observer la genèse de l'orientation verticale. Si l'on fait porter à un sujet des lunettes inversant sa vision du monde, «le paysage entier paraît d'abord irréel et renversé». Ensuite, «la perception normale commence à se rétablir», mais c'est le sujet qui se sent «la tête en bas`. Au bout d'une semaine, le sujet réussit progressivement à réorganiser son comportement visuo-moteur et à percevoir ‘normalement) à la fois l'orientation de son corps et

39. Merleau-Ponty: Phénoménologie, op.cit. 282.

40. Ibid., 282.

41. «Il faut, d'un côté, suivre le développement spontané du savoir positif, en nous demandant s'il réduit vraiment l'homme à la condition d'objet, et par ailleurs réexaminer l'attitude réflexive et philosophique, en recherchant si elle nous autorise vraiment à nous définir comme sujet inconditionné et intemporel. Peut-être ces recherches convergentes finiront par mettre en évidence [...], en deçà du sujet et de l'objet pur, [...] une troisième dimension», Merleau-Ponty: Titres et travaux, in: Merleau-Ponty: Parcours deux, Paris 2000, 13.

42. Cf. George Malcolm Stratton: Some preliminary experiments on vision without inversion of the retinal image, in: Psychological Review, 3 (1896), 611-617; Stratton: Vision without inversion of the retinal image, in: Psychological Review, 4 (1897), 361-360. Pour une présentation plus actuelle de ce paradigme d'expérimentation, cf. Jacques Paillard: Les déterminants moteurs de l'organisation de l'espace, in: Cahiers de Psychologie, 14 (1971), 261-316. 
l'orientation du monde..$^{43}$ Comment comprendre cette étonnante plasticité de la vision spatiale? Selon le schéma cartésien, deux réponses alternatives sont possibles: soit on cherche l'explication dans les contenus sensoriels, soit dans la pensée du sujet (ou, comme on dirait aujourd'hui, dans les (processus cognitifs`). Selon les termes de Merleau-Ponty, la première explication serait «empiriste», la deuxième «intellectualiste».

L'explication empiriste, adoptée par Stratton, part de l'idée qu'il faut distinguer entre deux sortes de données sensorielles: entre les données visuelles d'une part et les données tactiles ou kinesthésiques d'autre part. On voit la parenté avec la distinction que Descartes établit dans La dioptrique entre deux qualités premières: la «figure» et la «situation». ${ }^{44}$ Le port des lunettes provoque un conflit entre ces deux types de contenus sensoriels: l'image de la rétine, les sensations qui constituent le monde visuel, bascule de $180^{\circ}$, alors que le monde tactile et kinesthésique demeure «droit ${ }^{45}$ Intervient alors, selon Stratton, un facteur décisif: la perception kinesthésique du mouvement corporel contrôlé par la vue. Avant le port des lunettes, les sensations kinesthésiques provenant du mouvement que le sujet fait, pour par exemple atteindre ses pieds, était, dans l'ancien monde visuel, «associées» à la direction «vers le bas`. Maintenant, les kinesthèses sont, dans le monde actuel, vues comme un mouvement «vers le haut〉. L'expérience du corps propre permet donc, toujours selon Stratton, de repérer, de comparer et de réadapter les deux perceptions antagonistes, «en prenant, commente Merleau-Ponty, les données visuelles pour de simples signes à déchiffrer et en les traduisant dans le langage de l'ancien espace». Par répétition et «association», le monde visuel finit par rejoindre le monde kinesthésique, «la région du champ visuel où apparaissaient les pieds

43. Cf. Merleau-Ponty: Phénoménologie, op.cit., 282-283. Les gestes vont de nouveau à leur but, les sons émanant d'un objet visible seront bien localisés, et, surtout, les choses regagnent leur aspect consistant et réel.

44. On peut reformuler l'interprétation de Stratton en termes cartésiens: les «figures» des objets projetées sur la rétine sont inversées et entre en conflit avec la «situation» des objets qui, elle, demeure inchangée (car la situation, repérée par rapport aux sensations provenant des «nerfs insérés dans les muscles», ne dépend pas du port des lunettes de Stratton [Descartes: La dioptrique, op.cit., 60]. Mais si on peut formuler en termes cartésiens le conflit perceptif en question, la réadaptation de la vision globale de l'espace (englobant le visuel et le kinesthésique) est inconcevable; car pour Descartes, toutes ces correspondances réglées (entre les choses, leur figure et leur situation) sont «institué [e]s de la Nature» (ibid., 54). Nous y reviendrons.

45. Cela explique les perceptions irréelles et antagonistes au début de l'expérience: soit le sujet écoute son corps, le monde visuel semble alors inversé, soit il écoute les sensations visuelles, mais il va ressentir alors son corps «la tête en bas». 
du sujet cesse de se définir comme «le haut»». ${ }^{46}$ Toutefois, pour Merleau-Ponty, l'interprétation empiriste de Stratton est «inintelligible»: elle suppose que les directions «en haut et sen bas` soient «marqué[e]s dans les champs sensoriels par la distribution effective des sensations». Mais comment prendre l'orientation spatiale pour donnée avec les sensations, si l'expérience de Stratton montre précisément «que les mêmes contenus peuvent tour à tour être orientés dans un sens ou dans l'autre». ${ }^{47}$ Bref, l'expérience de Stratton est conçue pour étudier la genèse de l'orientation spatiale; on ne peut par conséquent, suivant cette approche généalogique, faire appel à une direction déjà constituée.

L'explication intellectualiste, l'autre volet cartésien, part également d'un espace déjà constitué, ne pouvant, elle non plus, rendre compte de l'émergence d'une nouvelle orientation spatiale. L'âme cartésienne, coupée de l'espace sensible, ne peut concevoir qu'un espace isotrope où toute direction est relative. C'est l'essence même de la mécanique du XVII ${ }^{\mathrm{e}}$ siècle qui sépare l'espace de l'expérience spatiale, neutralise son «interprétation naturelle», le conçoit sans point de vue. Le référentiel, l'axe des coordonnées géométriques, quels qu'il soit, sert à repérer les mouvements sans être repéré lui-même; pour le repérer, il faudrait un autre référentiel, et ainsi de suite: «La mise en place du monde est indéfiniment différée». ${ }^{48}$ Certes, le cogito, constituant l'espace, tient le pouvoir souverain d'inscrire toute direction dans l'espace, mais «il n'y a actuellement aucune direction et, par suite, aucun espace, faute d'un point de départ effectif, d'un ici absolu». ${ }^{49}$ Appliquant à l'homme les principes de la mécanique classique, introduisant en lui la scission entre corps-machine et âme désincarnée, toute conception cartésienne se prive de la possibilité de comprendre l'événement de polarisation spatiale. Cette impossibilité apparaît déjà très clairement dans La dioptrique de Descartes, notamment lorsqu'il s'apprête à définir la «situation» des objets, c'est-à-dire «le côté vers lequel est posée chaque partie de l'objet au respect de notre corps»..$^{50}$ D'abord, Descartes n'a pas d'autre possibilité que de supposer l'âme capable de sentir «en quel endroit est chaque partie du corps qu'elle anime» et de "transférer de là son attention à tous les lieux contenus dans les lignes droites qu'on peut imaginer être tirées de l'extrémité de chacune de ces parties, et prolongées à l'infini». ${ }^{51}$ Cette explication géométrique du «transfert d'attention» à partir d'un «là» repéré par

46. Merleau-Ponty: Phénoménologie, op.cit., $284 \mathrm{f}$.

47. Ibid., 285.

48. Ibid., 286.

49. Ibid.

50. Descartes: La dioptrique, op.cit., 59.

51. Ibid., je souligne. 
l'âme, cette "Géométrie naturelle», semble tout à fait vraisemblable. La peinture de la Renaissance qui construit la perspectiva artificialis s'appuie sur les mêmes principes géométriques pour «donner l'illusion» de la profondeur en trois dimensions. Descartes double ensuite l'explication géométrico-cognitive d'une explication causale: il suppose une correspondance réglée entre l'activité nerveuse (dans le corps) et l'apperception de la «situation» de chaque partie du corps (dans l'âme). Cette correspondance est «instituée de la Nature». ${ }^{52}$ Les difficultés d'un tel parallélisme entre la machine nerveuse et l'activité cognitive n'échappent toutefois pas à Merleau-Ponty. Dans L'œil et l'esprit, par exemple, où il fait une lecture critique de La dioptrique cartésienne, il se demande: «cet espace de son corps que [l'âme] étend aux choses, ce premier ici d'où viendront tous les là, comment le sait-elle?». ${ }^{53} \mathrm{~L}$ 'explication cartésienne ne fait que déplacer les questions: d'abord, les repères corporels («tous les là») ne sont que des repères secondaires, dérivés d'un ici primordial. Ensuite, comment comprendre l'institution d'une nouvelle orientation, démontrée par les expériences de Stratton et d'autres, si la nature avait fixé une fois pour toutes les rapports mécaniques entre le corps, l'espace et l'âme?

\title{
III. La genèse de l'espace brut
}

\author{
Le corps crée l'espace \\ comme l'eau crée le vase. \\ Tewfik el-Hakim \\ Quelque chose dans l'espace échappe \\ à nos tentatives de survol. \\ Maurice Merleau-Ponty
}

«Nous avons besoin d'un absolu dans le relatif»:54 cette formule résume les impasses de l'alternative cartésienne, tout en ouvrant une nouvelle perspective. Il s'agit de partir à la recherche d'une «troisième

52. «Cette situation est instituée de la Nature pour faire, non seulement que l'âme connaisse en quel endroit est chaque partie du corps qu'elle anime [...]; mais aussi qu'elle puisse transférer de là son attention à tous les lieux contenus dans les lignes droites qu'on peut imaginer être tirées de l'extrémité de chacune de ces parties, et prolongées à l'infini.» (Descartes: La dioptrique, op.cit., 59).

53. Merleau-Ponty: L'œil et l'esprit, op.cit., 53. La lecture de La dioptrique occupe tout le chap. III, 36-60.

54. Merleau-Ponty: Phénoménologie, op.cit., 287. 
spatialité»: un espace «qui ne glisse pas sur les apparences, qui s'ancre en elles [...], mais qui, cependant, ne soit pas donné avec elles à la manière réaliste, et puisse survivre à leur bouleversement». ${ }^{55}$

Il est donc nécessaire de revenir sur les dichotomies héritées du $\mathrm{XVII}^{\mathrm{e}}$ siècle: l'espace absolu, l'espace relatif; qualité première, qualité secondaire; contenu, forme; corps, âme. Quelles sont, en un mot, les difficultés de la dichotomie entre empirisme et intellectualisme? C'est que les deux conceptions parallèles partent du même «préjugé du monde»: ${ }^{56}$ toutes les deux supposent que l'espace, le corps et la pensée soient entièrement constitués, telles des réalités positives, données d'avance. Nul besoin par conséquent d' «interroger» l'expérience ellemême. ${ }^{57}$ Tout ce qui importe, c'est de rationaliser l'expérience, soit selon un mécanisme causal, soit suivant une logique préétablie, de préférence mathématique. Mais aucune des deux positions ne cherche dans un premier temps à décrire notre expérience spatiale telle qu'elle se fait, telle qu'elle nous apparaît, à l'état naissant, dans toute sa richesse. «Nul souci de coller à la vision»: faut-il alors s'étonner que la vision se comporte, selon la comparaison chère à Descartes, comme un aveugle? Inversement, si la vision était une mécanique aveugle, comment comprendre le don admirable que les aveugles peuvent développer à s'orienter dans l'espace urbain, à habiter l'espace?

\section{L'ÊTRE-AU-MONDE: LE CORPS DE LA PRATIQUE ET LES CHOSES QUI NOUS PARLENT}

L'orientation spatiale n'est donc pas une donnée, elle se constitue dans l'expérience; parfois elle émerge même brusquement, comme l'illustrent les «cas exceptionnels» dont abonde la Phénoménologie de la perception..$^{5}$ L'interprétation minutieuse d'une expérience du gestaltiste

55. Ibid.

56. Ibid., 34-40, 49-51, par exemple.

57. Cf. l'effort de Merleau-Ponty dans Le visible et l'invisible, ouvrage inachevé, de définir la phénoménologie comme une ontologie qui pratique une «pensée interrogative» (Merleau-Ponty: Le visible et l'invisible, Paris 1964). Pour une belle vue d'ensemble, Bernhard Waldenfels: Fragendes Denken, in: Waldenfels, Deutsch-französische Gedankengänge, Frankfurt/Main 1995, 159-171.

58. Nous ne pouvons naturellement pas présenter ici le nombre impressionnant d'études scientifiques que Merleau-Ponty discute. Dans le seul chapitre sur l'espace Merleau-Ponty analyse, dans une approche génétique, la vision avec distorsion des données visuelles (Stratton et de Wertheimer), les dessins ambigus (Koffka), l'induction de la perception de mouvement (Wertheimer), les pathologies de la perception spatiale (Goldstein), la spatialité dans le rêve, dans les hallucinations et schizophrénies (Fischer, Mayer-Gros et Stein, Binswanger, Minkowski). 
Wertheimer va permettre à Merleau-Ponty de réinterpréter Stratton afin de formuler un nouveau rapport entre l'homme, son corps et l'espace. L'expérience de Wertheimer fait de nouveau intervenir un instrument optique: le miroir, objet de plus en plus «magique» aux yeux de Merleau-Ponty ${ }^{59}$ pour une raison paradoxale, j'y reviendrai. Dans le scénario expérimental, le sujet se trouve dans une chambre qu'il ne peut voir que par l'intermédiaire d'un miroir. Seul dispositif galiléen: le miroir «reflètes la chambre en l'inclinant de $45^{\circ}$ par rapport à la verticale. ${ }^{60}$ Quelle est alors l'expérience de cet espace miroité?

«Le sujet voit d'abord la chambre oblique. Un homme qui s'y déplace semble marcher incliné sur le côté. Un morceau de carton qui tombe le long du chambranle de la porte paraît tomber selon une direction oblique. L'ensemble est très 〈étranges.»

Cette scène rappelle les scènes d'une inquiétante étrangeté dans lesquelles nous entraînent les tableaux de M.C. Escher, tel Relativité de 1953, où plusieurs orientations spatiales opposées coexistent dans un même espace. Mais alors qu'il nous est impossible de réconcilier les perspectives «incompossibles» ${ }^{61}$ qui coexistent dans le même tableau d'Escher et de réduire à un espace uniforme ses univers polymorphes, un évènement surprenant survient dans l'expérience de Wertheimer: après quelques minutes seulement, et sans que le sujet ait la possibilité d'une exploration motrice, la chambre perçue dans le miroir revient «brusquement» à la verticale, l'homme se remet à marcher horizontalement et le carton chute de haut en bas, comme la pierre d'Aristote. Le 〈haut et le 〈bas` se sont instantanément redistribués. Comment comprendre cet événement? Notons tout d'abord que l'explication causale est exclue. Aucun paramètre pris isolément ne peut être considéré comme responsable de la brusque redistribution spatiale. L'exploration motrice, c'est-à-dire les sensations kinesthésiques que Stratton considérait comme paramètre déterminant est contrôlée dans l'expérience de Wertheimer, le sujet n'ayant pas le droit de quitter sa place. ${ }^{62} \mathrm{Il}$ ne s'agit pas d'un acte conscient, «thétique» du sujet percevant qui comparerait les données pour les déchiffrer correctement, comme si

59. Cf. notamment le tout dernier texte de Merleau-Ponty: L'œil et l'esprit, op.cit., 33-35, et, contre Descartes, 37-39.

60. Cf. Wertheimer: Experimentelle Studien über das Sehen von Bewegung, Zeitschrift für Psychologie, 60 (1912), 258.

61. ¿Incompossible au sens leibnizien: les perspectives ne sont pas contradictoires en soi, mais leur coexistence dans le même monde n'est pas possible. Cette idée devient de plus en plus important pour Merleau-Ponty, comme nous le verrons.

62. Bref, ni le visuel, ni le kinesthésique, ni leur conflit, ni leur mise en rapport causale ou cognitive ne peuvent expliquer l'évènement de la réorientation spatiale. 
c'étaient des signes. Il s'agit d'un évènement global qu'on ne peut comprendre que comme un événement de réorganisation spontanée de l'ensemble du champ perceptif et motrice: «le champ perceptif se redresse [...] parce que je vis en lui, parce que je me porte tout entier dans le nouveau spectacle et que j'y place [...] mon centre de gravité». ${ }^{63}$

Que la chambre soit perçue comme oblique ou comme droite, dans les deux cas l'espace est orienté. Il convient donc de partir, conclut Merleau-Ponty, d'un «certain niveau spatial» qui donne l'orientation. C'est par rapport à ce niveau que le spectacle expérimental apparaît d'abord oblique, ensuite droit. Ce niveau n'est déterminé ni par les contenus visuels, ni par l'orientation du corps propre, même si les deux contribuent sans doute à la constitution du niveau. ${ }^{64}$ Comment le niveau est-il institué, comment peut-il basculer? Nous l'avons vu, si l'on sépare, à la manière de la mécanique classique ou du cartésianisme, le sujet d'avec le monde dans lequel il est installé, notre expérience quotidienne de l'espace comme étant orienté devient incompréhensible.

Mais en réalité, nous ne sommes pas dans l'espace comme un objet. Nous habitons l'espace. Depuis toujours, nous marchons dans l'espace, nous y manions des objets, nous y rencontrons autrui. C'est en apportant son niveau spatial habituel que le sujet de Wertheimer cherche à s'installer dans la chambre perçu dans le miroir. C'est par rapport à ce niveau habituel que le spectacle expérimental apparaît d'abord oblique. Mais l'espace ainsi orienté est «étrange», «irréel». Il devient difficilement habitable. Se produit alors l'évènement qui réinstalle le sujet de manière plus cohérente et plus entière dans la chambre: le spectacle expérimental «induit» un autre niveau, de sorte qu'il apparaisse de nouveau droit. Toutefois, si le sujet habite l'espace, s'il réussi à s'installer dans un espace inhabituel avec son corps parce qu'il cherche toujours à habiter l'espace, son corps n'est pas un corpsobjet qui serait comme une chose dans l'espace, mais un «corps virtuel», un corps de la pratique déterminé par ses projets:

«ce qui importe pour l'orientation du spectacle, ce n'est pas mon corps tel qu'il est en fait [...], mais mon corps comme système d'actions possibles, un corps virtuel dont le «lieu» phénoménal est défini par sa tâche et sa situation». ${ }^{65}$

Le monde, de son côté, n'est pas l'espace physique ou géométrique, les choses ne sont pas des simples objets dénués de tout sens qui ne feraient qu'occuper une place géométrique abstraite. Les choses ont un

63. Merleau-Ponty: Phénoménologie, op.cit., 290.

64. Ibid., $287 f$.

65. Ibid., 289, je souligne. 
sens pour nous, elles nous parlent. Elles nous sollicitent parce que nous les manions, que nous vivons avec elles, selon elles. ${ }^{66}$ Le sujet peut habiter l'espace parce qu'il est incarné, les choses nous parlent parce que notre corps leur répond.

Ce que la description de Merleau-Ponty fait apparaître progressivement est un rapport de solidarité entre le sujet et son corps, entre les choses et l'espace, un «être-au-monde», "véhiculé» par le corps propre. ${ }^{67}$ Cette pensée de la solidarité se situe en deçà du cartésianisme. En fait, le cartésianisme consiste à couper les liens intimes qui se tissent entre l'homme et le monde, à définir l'homme et le monde comme deux substances positives et indépendantes: res extensa et res cogitans. Une telle pensée de la séparation, dont le doute radical et la certitude mathématique ne représentent que deux faces du même divorce d'avec la réalité, ne peut rétablir le rapport entre l'homme et le monde que de deux manières: soit en construisant un rapport d'extériorité mécanique, partes extra partes, soit en postulant un rapport d'intériorité absolue où le sujet souverain constitue le monde, lui donne un sens, sans être dérangé par son corps. On coupe pour recoudre.

Merleau-Ponty, en revanche, décrit la solidarité primordiale comme un rapport de «motivation», dont la causalité mécanique et la constitution transcendantale ne sont que des dérivés abstraits: les choses dans le monde offrent une vision, proposent un sens, sollicitent une réaction; l'homme, à son tour, y répond, assume une décision, lit dans la texture du visible. Cela signifie que «la relation du motivant et du motivé est $[. .$.$] réciproque». { }^{6}$

Dès lors, le changement soudain de l'orientation spatiale dans

66. Ainsi, dans l'expérience de Wertheimer «tout se passe comme si certains objets (les murs, les portes, le corps de l'homme dans la chambre) [...] prétendaient de soi à fournir les directions privilégiées, attiraient à eux la verticale.» Merleau-Ponty: Phénoménologie, op.cit., 287-288. La compréhension merleau-pontienne de l'objet pratique est proche ici de ce que Heidegger décrit comme Zeug (que Vezin traduit par l'ancien mot «util» pour lui enlever la connotation technique), cf. Martin Heidegger: Sein und Zeit, Tübingen 1927 (trad. fr. par François Vezin, Paris 1986).

67. Merleau-Ponty: Phénoménologie, op.cit. 323f., mais aussi 97f., 150f., 161f., 217f., 270-272, 394f., 404f., 414f., 450f., 491-494, 503-505. On peut rapprocher, bien sûr, «l'être-au-monde» au sens de Merleau-Ponty à l'idée de Heidegger de «Inder-Welt-sein», mais à condition de ne pas oublier, chez Merleau-Ponty, la richesse des descriptions et la pénsée originale du corps propre.

68. Merleau-Ponty: Phénoménologie, op.cit., 399-400. L'idée de la motivation, déjouant la pensée dichotomique de la causalité et de la constitution, traverse toute la Phénoménologie de la perception; cf. par exemple, 39f. (attention et jugement), 56-63 (motivation), 198 (sexualité), 201f., 299f. (espace, profondeur), 356, 417f., 488 (temporalité). 
l'expérience de Wertheimer est motivé par la possibilité d'un autre rapport plus intime entre le sujet et le monde dans lequel il s'installe. La nouvelle spatialité surgit quand le sujet et le monde se recroisent, dans une sorte de «chiasme»: il y a orientation spatiale quand

«entre mon corps comme puissance de certains gestes, comme exigence de certains plans privilégiés, et le spectacle perçu comme invitation aux mêmes gestes et théâtre des mêmes actions, s'établit un pacte qui me donne jouissance de l'espace comme aux choses puissance directe sur mon corps». ${ }^{69}$

\section{Le MYSTÈre de LA PROFONDEUR ET L'ESPACE CLASSIQUe DE L'ART PICTURAL}

La question de notre orientation dans l'espace se pose traditionnellement, nous l'avons vu, en termes alternatifs d'objectivité ou de subjectivité. L'orientation verticale semble surtout être déterminée par des facteurs objectifs, tels la gravitation conditionnant les kinesthèses ou l'axe vertical du corps propre. Merleau-Ponty montre cependant, en développant les résultats des expériences gestaltistes, qu'il n'est pas possible de réduire la perception de la verticalité à ces données objectives. Même la verticalité, la plus objective des trois dimensions, ne dérive pas de l'espace mécanique. Elle naît du «chiasme» entre le sujet enraciné dans le monde et le monde habité par le sujet incarné.

Quant à la dimension de la profondeur, elle semble encore moins être déterminée par des données objectives. Si je peux bien appréhender visuellement l'intervalle entre deux chaises en face de moi (la largeur), si je peux bien apercevoir le vase sur la table ou la pierre qui tombe vers le bas, tout en entrevoyant l'axe défini par ma tête et mes pieds (la hauteur), la profondeur n'est pas directement donnée avec les objets perçus: la projection visuelle sur nos rétines est bidimensionnelle, le bâton que l'aveugle cartésien tend dans la profondeur peut se contracter en un point. Bref, vues en profondeur, les choses risquent de se cacher les unes les autres et de masquer ainsi la profondeur. Si la philosophie et la physique classiques définissent l'espace par la simultanéité, cette définition ne semble pas valable pour la profondeur: la même coexistence simultanée des choses qui donne à voir la largeur et la hauteur dissimule la profondeur. La profondeur a ainsi «quelque chose de paradoxal», quelque chose d'un «mystère»: ${ }^{70}$ elle m'apparaît parce que la vision se fait depuis mon corps auquel je suis «collé» et que je vois des objets l'un derrière l'autre; mais, du

69. Merleau-Ponty: Phénoménologie, op.cit., 289, je souligne.

70. Merleau-Ponty: L'œil et l'esprit, op.cit., 45. 
même coup, elle se dérobe à mon regard, puisque je ne peux jamais quitter mon corps qui m'enracine dans l'espace, pour l'embrasser du regard.

Quitter le corps propre et la terre comme centre spatial: c'est précisément ce désir qui anime les conceptions mécaniques du XVII siècle. On y parvient par l'abstraction imaginative, par l'expérience de pensée galiléenne et la construction géométrique cartésienne. On fait voler des papillons imaginés dans un référentiel quelconque. On persuade la vision naturelle de se détacher de son exercice effectif, de quitter son espace, pour la transformer en pensée mécanique de la vision. On compare la vision à un aveugle sagace. Ainsi la vision de la profondeur n'a-t-elle plus de mystère: les conceptions classiques de la profondeur, qu'elles soient empiristes ou intellectualistes, partent de l'idée que nous ne voyons pas la profondeur, mais que nous la reconstruisons. La 'grandeur apparentes ou le degré de 'convergence des yeux' permettrait, selon une trigonométrie cognitive ou neuronale, que Descartes appelle "Géométrie naturelle», de calculer la distance entre l'objet et moi. La profondeur ne serait pas vue, mais «connue». ${ }^{71}$

Pourtant, une fois de plus, l'explication est circulaire et présuppose l'espace constitué dont il s'agit de comprendre la genèse. Je ne peux déchiffrer les signes visuels (grandeur apparente et convergence) qu'à condition de connaître le code géométrique, qu' «à condition d'insérer mes yeux, mon corps et l'extérieur dans un même espace objectif». Pour construire cet espace objectif, il faut que le sujet quitte le lieu où il est installé, son point de vue sur le monde, qu'il se positionne latéralement afin de voir la distance, non pas en profondeur, mais en largeur. Or, supposer que la «profondeur telle qu'elle s'offre à moi» et la «profondeur pour un spectateur placé latéralement» soient équivalentes, c'est, de nouveau, assimiler l'espace vécu à l'espace physique isotrope. ${ }^{72}$ Ce n'est plus la profondeur que je vois depuis un <ici absolù que m'ouvre à un 〈là-bas»; c'est une simple distance que je peux mesurer en "survolant» l'espace, 〈ici et 〈là-bas〉 devenus interchangeables. Cependant, la généalogie de la profondeur, de même que celle

71. Plus l'objet s'éloigne, plus son image paraît petite; plus l'objet s'approche, plus les yeux convergent. De nouveau, Descartes fait appel à «notre aveugle» et le repère dérivé «là»: «comme notre aveugle, tenant les deux bâtons $A E, C E[\ldots]$ et sachant seulement l'intervalle qui est entre ses deux mains $A$ et $C$, et la grandeur des angles $\mathrm{ACE}, \mathrm{CAE}$, peut de là, comme par une Géométrie naturelle, connaître où est le point E» (Descartes: La dioptrique, op.cit., 62, je souligne). Ce principe cartésien soustend toujours les explications cognitivistes de la perception de la profondeur.

72. Merleau-Ponty: Phénoménologie, op.cit., 297. 
de la verticalité, nous demande de rejeter ce préjugé d'un espace constitué et d'un sujet qui les survole comme un être divin. ${ }^{73}$

La psychologie de la Gestalt a mis en évidence que les signes objectifs de la profondeur, telle la grandeur apparente, n'apparaissent pas dans le champ perceptif, dans la vision effective. ${ }^{74}$ Pour apercevoir la grandeur apparente, il faut prendre des mesures artificielles. Ainsi, si je veux comparer la grandeur apparente de la lune à celle d'une pièce de monnaie, je dois fermer un œil, placer la pièce sur la ligne de projection décrite par le bâton cartésien, la tenir à la bonne distance, pour qu'elle recouvre la lune. Mais ce faisant j'ai démembré le champ perceptif, j'y ai isolé la lune et la pièce pour faire apparaître leur grandeur apparente, je les ai rendues irréelles, je ne les vois plus dans l'espace, là où elles me sont apparues initialement. De la sorte, «j'ai changé les proportions du spectacle» et «j'ai réduit la perspective vécue à la perspective géométrique». ${ }^{75} \mathrm{Si}$ j'ouvre pourtant mon deuxième œil, les grandeurs apparentes disparaissent et j'aperçois à nouveau les deux ‘objets` dans l'espace. La lune et la pièce de monnaie, telles que la vision les donne, sont incommensurables, même si je peux par ailleurs comparer leur grandeur apparente. Ils m'apparaissent, dit MerleauPonty, comme un «objet-grand-vu-de-loin» et un «objet-petit-vu-deprès». ${ }^{76}$

Et pourtant, l'art pictural de la Renaissance, avec des visionnaires tels Brunelleschi, Uccello, Alberti, de Vinci, Dürer n'est-il pas parvenu, pour la première fois d'une manière naturaliste, à nous faire voir les choses en profondeur? Son art ne consiste-t-il pas précisément à projeter la «perspective vécue» dans la "perspective géométrique» pour construire une perspective d'illusion? Cette perspectiva artificialis, n'est-elle pas la plus réaliste, puisqu'elle reproduit à merveille la perspectiva naturalis, la projection sur nos rétines des choses et de leurs situations spatiales, telle qu'elle est décrite dans La dioptrique? En effet, trois siècles avant Descartes, l'Annonciation des frères Lorenzetti démontre l'efficacité de la représentation spatiale par la perspective centrale. Le sol en échiquier de cette représentation est conçu avec un seul point de fuite. Même si la construction entière du tableau n'est pas encore parfaite, ${ }^{77}$ notre regard est irrésistiblement attiré dans la pro-

73. Ibid., 295-296.

74. Kurt Koffka: Some Problems in Space Perception, in: C. Murchison: Psychologies of 1930, Worcester 1930, 161-187.

75. Merleau-Ponty: Phénoménologie, op.cit., 301.

76. Merleau-Ponty: La prose du monde, Paris 1969, 73.

77. Les diagonales que l'on peut tracer entre les carreaux se transforment en courbes alors qu'elles devraient être droites; le traditionnel fond d'or subsiste, limite et bouche l'espace; et les personnages flottent au-dessus de la dalle en échiquier. Est-ce 
fondeur de l'espace peint, nous pouvons même saisir les dimensions des objets représentés et les distances qui les séparent. Comme le résume Panofsky dans La perspective comme forme symbolique, ce tableau «représente [...] le premier exemple d'un système de coordonnées qui, dans une sphère du concret artistique, rend l'espace systématiques moderne matériellement visible». ${ }^{78}$ Par rapport à la perspective hiérarchique du Moyen Âge, et même par rapport aux constructions plus complexes de Duccio et de Giotto, cette conception semble constituer un immense pas en avant vers une représentation unifiée et cohérente de l'espace objectif. ${ }^{79}$

Toutefois, cette historiographie de l'art qui présente la découverte de la perspective centrale comme un progrès univoque et linéaire s'expose à une vision extrêmement réductrice - tout aussi simpliste que le portrait de l'histoire de la mécanique critiqué par Feyerabend, que j'ai cité au début de ce travail. ${ }^{80}$ En effet, on a pu montrer, d'une part, que la perspective centrale ne fut pas une invention ex nihilo du grand architecte florentin Brunelleschi, mais «le dernier acte d'un processus historiquement vérifiable dans ses phases successives». ${ }^{81} \mathrm{On}$ a pu montrer, d'autre part, que la perspective courbe des Anciens continue à

des imperfections ou bien des indices d'une volonté d'expression, d'une «forme symbolique» (Cassirer, Panofsky)?

78. Erwin Panofsky: La perspective comme forme symbolique, Paris 1975, 125.

79. La Cène du retable du maître-autel (1301-1308) de Duccio di Buoninsegna avec un plafond en perspective constitue certes une représentation spatiale. Mais cette spatialité est encore incohérente en ce sens que les lignes de fuite ne convergent pas systématiquement vers un point, que les objets, par exemple la table de la Cène, «ne semble pas être à l'intérieur, mais en avant de cette 〈boîte d'espace»» et que la partie centrale et les autres parties ne sont pas construite selon le même schéma perspectif (cf. Panofsky: La perspective, op.cit. 120-123).

80. De même que la pensée galiléenne et cartésienne, aussi profitable qu'elle soit, nous éloigne de notre expérience naturelle, la perspective centrale fige notre vision vivante. De même que l'interrogation aristotélicienne de l'expérience spatiale reste signifiante pour une phénoménologie de l'espace, l'espace sphérique de l'Antiquité reste valide pour la pratique de la perspective picturale, dans la mesure où il rend compte, contrairement à la projection plane, de la concavité de l'image rétinienne et des effets produits par le mouvement des yeux, comme le rappelle Panofsky (cf. Panofsky: La perspective, op.cit., 49-67).

81. Cf. Marisa Dalia Emiliani: La question de la perspective, in: Panofsky: La perspective, op.cit., 24. Un des premiers à s'interroger sur la continuité historique selon laquelle la perspective est découverte est Guido Hauck: Die subjektive Perspektive, Stuttgart 1879. 
mener une existence en pleine Renaissance ${ }^{82}{ }^{2}$ 'histoire de la perspective n'a pas débuté avec la Renaissance et ne s'est pas arrêtée à cette époque. ${ }^{83}$ Ainsi, dans la seule période de 1450 à 1550 , différents procédés coexistent pour construire la perspective, procédés qui combinent de diverses manières l'espace sphérique des Anciens (la perspectiva naturalis conçue comme angulaire) et l'espace isotrope des Modernes (la perspectiva artificialis plane), deux conceptions de l'espace qui ne semblent pas être conciliables. Ces incohérences témoignent d'une véritable quête de la profondeur vivante, telle qu'elle se montre dans notre perception naturelle. Léonard de Vinci, par exemple, imagine une représentation où les choses figureraient seulement à l'image, non pas de la grandeur projetée selon la construction d'Alberti, mais de leur grandeur apparente perçue. Cela autorise une vision grande angle, une perspective panoramique, contrairement au regard par une «fenêtre» qu'imagine Alberti. ${ }^{84}$ Il trouve d'une part des "raccourcis naturels» selon la perspective angulaire pour les grandeurs perçues sur les parties latérales du tableau (pour la largeur), alors que d'autre part, il constate "per isperienza» un rapport proportionnel pour la grandeur apparente du même objet s'éloignant du spectateur (pour la profondeur), ce qui correspond à la perspective plane. ${ }^{85}$ L'espace sphérique pour la largeur, l'espace isotrope pour la profondeur: n'est-ce pas faire coexister deux grammaires visuelles, géométriquement incompatibles, dans le même espace? En effet, dès la Renaissance, les prétentions réalistes de la perspective centrale sont l'objet d'une vive polémique. S'affrontent alors théoriciens et praticiens, l'induction et la déduction, en quête de la perspective idéale, uniforme et unique, de la costruzione legittima qu'il s'agit d'arracher à la foule des techniques pratiquées. ${ }^{86} \mathrm{Il}$ peut arriver qu'un peintre-géomètre reproche, au nom d'un théorème central, à un futur premier peintre du Roi une faute de perspective

82. Panofsky en trouve un exemple frappant dans un traité anonyme du XVI. siècle: Les règles du Dessin (cf. Emiliani: La question de la perspective, op.cit., 28).

83. De même que l'histoire de l'espace physique a continué avec Mach, Einstein et d'autres, sans se clore jusqu'à aujourd'hui, l'histoire de la perspective a connu une renaissance avec Cézanne, Braque, Picasso, Chirico, Hockney et bien d'autres.

84. «Mon premier acte [...] est de tracer un rectangle [...] en guise de fenêtre ouverte par où je puisse voir le spectacle [historia]», Leon Battista Alberti: Della Pittura, 1435, (trad. fr. par J.-L. Schefer, Paris 1992, livre I, §19.)

85. Cf. Panofsky: La perspective, op.cit. 62-63.

86. La 〈costruzione legittima〉 d'Alberti, première systématisation, est suivie des méthodes de Piero della Francesca, de Léonard, de Viator, de Dürer, de Cousin, de Vries, de Barbaro et de Desargues. Cf. par exemple la tentative de synthèse chez Guidobaldo del Monte: Perspectivae libri sex, Pesaro 1600 (facsimilé, Rome 1984). Ce géomètre seul présente 23 méthodes principales. 
dans le tracé des ombres. ${ }^{87} \mathrm{Si}$, au $\mathrm{XV}^{\mathrm{e}}$ siècle, la perspective est un modèle fécond pour ceux qui cherchent à savoir ce qu'ils voient, elle devient, au début du XVII ${ }^{\mathrm{e}}$ siècle, l'encombrant privilège de ceux qui cherchent à voir ce qu'ils savent.

\section{L'ESPACE BRUT: INCOMPOSSIBILITÉ ET SIMULTANÉTTÉ «SAUVAGE» DES CHOSES}

Les techniques de la perspective «ont encouragé la peinture, constate Merleau-Ponty, à produire librement des expériences de profondeur [...]», mais elles étaient fausses, quand «elles prétendaient clore la recherche et l'histoire de la peinture, fonder une peinture exacte et infaillible». ${ }^{88}$ Ce constat témoigne d'un mode d'interrogation propre à la phénoménologie de Merleau-Ponty: revenir sur les objectivations et les idéalisations de notre expérience tout en traversant précisément les champs de recherches qui l'objectivent et l'idéalisent. Ainsi MerleauPonty cherche-t-il à mettre au jour le lien primordial entre l'homme et l'espace qu'il habite dans les expérimentations auxquelles la psychologie de la Gestalt soumet ses sujets. Il cherche à expliciter le sens phénoménologique et ontologique que les expériences de Stratton, de Koffka et de Wertheimer comportent implicitement. De même, la géométrisation de l'espace opérée par l'optique cartésienne et par la perspective classique peut nous enseigner le sens ontologique de la profondeur:

«il fallait d'abord idéaliser l'espace, concevoir cet être parfait [...], clair, maniable et homogène, $[\ldots]$ pour qu'on pût un jour trouver les limites de la construction, comprendre que [...] les dimensions sont prélevées par les divers métriques sur une dimensionnalité, un Être polymorphe, qui les justifie toutes sans être complètement exprimé par aucune.» (ibid., 48)

Pourquoi l'expérience de la profondeur, l'être de l'espace ne se laisse pas exprimer complètement par les techniques de la perspective? Pourquoi la «fenêtre» qu'Alberti a ouverte ne donne pas sur le paysage tel qu'il nous apparaît? Que dit cette impossibilité de l'être de l'espace? $C^{\prime}$ est que la perspective géométrique fige la dynamique perceptive du Dasein, de cet être-au-monde qu'est l'homme. Elle arrête cette pratique

87. C'est le reproche qu'Abraham Bosse fait à Le Brun, pour défendre son maître Girard Desargues. Le théorème de Desargues prouve la possibilité de ramener à deux dimensions toute configuration spatiale et permet d'unifier la multitude de procédés graphiques pratiqués à l'époque. Mais a-t-il pu clore de la sorte la recherche de la profondeur? Cf. René Taton: L'œuvre mathématique de Desargues, Paris 1981.

88. Merleau-Ponty: L'œil et l'esprit, op.cit., 49. 
dynamique qu'est la vision, pratique d'un être qui est au milieu des choses et qui les voit parce qu'il les manie, englobé par l'espace qui surgit dans cette promiscuité. La perspective centrale n'est parfaite que pour un spectateur qui accepte de se transformer en une sorte de cyclope, de se placer à l'endroit prévu par la construction, sans le droit de bouger ni le corps ni la tête ni l'œil. Toutefois, même lorsque je reste immobile, mes yeux ne cesse de parcourir le spectacle et de se retrouver dans l'espace actuel, attirés par les qualités et les tracés qui me sollicitent, multipliant les perspectives qui m'ouvrent à la profondeur. Je ne contemple pas le monde à travers la fenêtre d'Alberti ou le sténopé de Brunelleschi photographe; je suis situé au milieu des visibles qui m'entourent, j'en fais partie, je suis «du même étoffe», ${ }^{89}$ puisque je suis moi-même un corps visible. Même lorsque je regarde par la fenêtre, la fenêtre elle-même se situe dans l'espace qui n'est pas devant moi, mais qui m'enveloppe.

L'artifice de la perspective consiste à attribuer à chaque chose sa place déterminée, à créer un espace où les choses coexistent paisiblement dans une simultanéité géométrique. En réalité, les choses, en même temps qu'elles se donnent à voir, se dérobent de multiples façons à mon regard. Qu'est-ce que je vois au juste quand j'embrasse d'un seul regard un bouquet dans un vase placé sur une table, â côté des pommes et devant une assiette, telle la nature morte Le vase bleu de Cézanne ${ }^{90}$ ? Simplement, je ne peux regarder qu'un objet à la fois. Sans que pour autant disparaissent les autres objets. Si mon regard fixe le bouquet, le vase, les pommes et l'assiette sur la table apparaissent en marge, restent visibles. Si mon regard fixe le vase (ou les pommes ou... le mur visible au fond de ce tableau, l'embrasure d'une ouverture sans porte qui donne sur un fragment de ciel et de prairie...), à chaque fois, les autres objets vont devoir reculer en arrière-plan. La chose perçue actuellement, nous apprend la psychologie de la Forme, se fait Gestalt en se dessinant sur le fond des autres. Les choses coexistent donc bien dans mon regard, mais jamais au même titre. Il y a «rivalité» entre elles. ${ }^{91}$ Elles se disputent mon regard: celles qui sont en marge ne cessent de solliciter mon regard, de revendiquer mon attention, aspirant à une présence pleine; celle que je regarde ne cesse de me préoccuper, de me révéler des nouveaux aspects, de se déployer dans le temps et dans l'espace de sorte que je ne puisse jamais arrêter ou épuiser sa visibilité. Il y a ainsi deux horizons indéfinis: l' «horizon extérieur» des choses perçues en marge qui attendent d'être regarder pleinement et l' $\mathrm{l}^{\prime}$ horizon intérieur» de la chose que mon regard fixe,

89. Merleau-Ponty: L'œil et l'esprit, op.cit., 21.

90. Nature morte de 1885-1887, Paris, Musée du Louvre.

91. Cf. Merleau-Ponty: La prose du monde, op.cit., 73-74. 
sans pouvoir arrêter son déploiement spatial. ${ }^{92} \mathrm{C}^{2}$ est parce que l'apparition de l'objet est inépuisable que l'objet est là, dans toute sa vivacité, voluminosité, consistance, épaisseur. La perspective a ceci d'artificiel qu'elle m'oblige d'arrêter ce spectacle dynamique et inépuisable des visibilia. Elle m'empêche de répondre aux sollicitations des choses. Elle neutralise leurs conflits et, du même geste, elle sépare les choses qui sont intimement liées dans leur émulation, pour les disposer, partes extra partes, dans un espace purifié de toute querelle : «chaque chose cesse alors d'appeler sur soi toute la vision, fait aux autres des concessions et consent à n'occuper plus sur le papier que l'espace qui lui est laissé par elles». ${ }^{93}$

Que signifie donc de dire qu'une chose est dans l'espace, qu'elle est là? C'est parce que je suis enraciné ici que la chose apparaît là-bas. Il n'y aurait pas d'apparition du phénomène dans l'espace sans cet éloignement primordial et irréductible. L' ‘ici signifie mon appartenance aux choses perceptibles, l'enracinement originaire de moi qui suis corps-pratique en l'espace englobant. Il est un lieu unique, absolu, même si (ou justement parce qu') il peut toujours changer:

«L'espace n'est plus celui dont parle la Dioptrique, réseau de relations entre objets, tel que le verrait un tiers témoin de ma vision [...], c'est un espace compté à partir de moi comme point ou degré zéro de la spatialité. Je ne le vois pas selon son enveloppe extérieur, je le vis du dedans, j'y suis englobé». ${ }^{94}$

La terre originaire de l'expérience ne se meut pas, dit Husserl selon Aristote, aux antipodes de Copernic, Galilée et Descartes. Je peux certes toujours explorer davantage ce que je vois, m'approcher, tourner l'objet, mais toujours au prix des aspects de l'objet qui se déroberont, précisément parce que je m'approche, que je regarde de plus près. Lorsque je regard un détail, je perd la vue d'ensemble, lorsque je regarde le dos, je perd de vue la face, et vice versa. L'horizon recule sans cesse et les choses «fuient dans un éloignement que nulle pensée ne

92. Je peux, en fixant le bouquet, regarder de plus près les fleurs rouges, ou les blanches...; je peux remarquer un bleu particulier qui se dessine sur d'autres bleus, découvrir que les pétales blancs ne sont pas vraiment blancs..., tout en entrevoyant au fond la table, l'assiette...

93. Merleau-Ponty: Le langage indirect et les voix du silence, in: Merleau-Ponty: Signes, Paris 1960, 62. Bien évidemment, la construction en perspective centrale ne m'empêche pas pour autant de me placer autrement que prévu par le «centre de vision» et de laisser libre jeu à mon regard, qui découvrira, paradoxalement, à la fois le paysage ordonné en perspective et le spectacle des objets qui se disputent mon regard.

94. Merleau-Ponty: L'œil et l'esprit, op.cit., $58 f$. 
franchit». ${ }^{95}$ Que la chose soit là-bas signifie qu'elle n'est pas à une distance mesurable à vol d'oiseau entre elle et moi, son «là-bas` n'est pas l'envers d'un autre <icis: nos situations ne sont pas interchangeables. Le 〈là-bas` n'est pas une place dans un contenant spatial, mais apparition de la chose $\mathrm{du}$ fond des autres, avec des faces visibles et des faces cachées, dans un éloignement irréductible et une proximité toujours reportée. Ainsi la profondeur n'est-elle pas une des trois dimensions de mesure, mais la dimensionnalité primordiale même. Le lieu du phénomène est la profondeur que creuse son apparition.

C'est pourquoi la perspective centrale ne peut être qu'un moyen d'expression, une «forme symbolique» parmi d'autres, pour capter la dynamique spatiale de la vision et des choses. C'est pourquoi l'investigation de la spatialité et l'histoire de la peinture en quête de la profondeur ne sauraient s'achever. Merleau-Ponty cite Giacometti: «Moi je pense que Cézanne a cherché la profondeur toute sa vie». ${ }^{96}$ C'est pourquoi les peintres font souvent coexister plusieurs logiques de perspective dans un et même tableau, multipliant les points de vue, provoquant une tension entre les choses représentées - d'ailleurs déjà à l'époque de la Renaissance et avant. Giotto, par exemple, emploie tout à la fois la perspective primitive, la perspective parallèle et une perspective qui se rapproche de la perspective d'illusion pour réaliser les fresques de la Vie de saint François. Jan van Eyck, dans Les époux Arnolfini, peint des arêtes de la fenêtre de gauche et celle du lit avec des points de fuite différents, l'ensemble est composé à partir de plusieurs points de vue. L'artiste a vraisemblablement utilisé un appareil optique puis a effectué un collage, déjouant ainsi la perspective centrale qu'il maîtrisait par ailleurs parfaitement. Le spectateur qui observe une telle scène semble être mobile. Cézanne déjoue l'opposition entre la qualité première et la qualité secondaire, entre la forme et le contenu, en cherchant le volume des choses et la profondeur de l'espace dans la couleur et dans la physionomie du tracé qui n'est pas la «ligne prosaïque» de la projection géométrique. «C'est donc ensemble qu'il faut chercher l'espace et le contenu». ${ }^{97}$

Il y a donc bien simultanéité des choses dans l'espace, mais elle ne signifie pas égalité d'existence ni cohabitation arrangée. Ce n'est pas la simultanéité géométrique des objets entièrement constitués, indifféremment étendus dans un espace vide; c'est une simultanéité «sauvage», où chaque chose «revendique une présence absolue qui est incompossible avec celle des autres». ${ }^{98}$ L'incompossibilité, tournure leib-

95. Ibid., 50.

96. Ibid., 64.

97. Ibid., 66 et les pages 67-79 sur la couleur et la ligne, sur Cézanne et Klee.

98. Merleau-Ponty: Signes, op.cit., 228. 
nizienne réinterprétée par Merleau-Ponty, ne veut pas dire contradiction logique, ni impossibilité réelle de coexistence, mais signifie le fait pratique que nous ne pouvons percevoir ou faire quelque chose qu'au prix d'autres. Ce fait pratique est pour Merleau-Ponty un fait absolu, ontologique: on ne saurait mettre le jeu entre éloignement et approchement, entre clarté et obscurité, entre visible et invisible au compte de la finitude de l'être humain. L'éloignement et la profondeur sont des dimensions originaires de l'Être, qui n'est pas une entité positive et constituée, qui ne se dévoile que dans une différenciation négative.

\section{Conclusion}

On ne peut étudier que ce qu'on a d'abord rêvé.

Gaston Bachelard

La question de l'espace révèle de façon exacerbée les difficultés que rencontre toute pensée dualiste, dont le nom de Descartes est devenu l'emblème. En déracinant l'homme du monde qu'il habite, cette pensée se retrouve face à une alternative peu satisfaisante: soit on conçoit l'espace selon les rapports mécaniques postulés entre les objets dépouillés de leur sens et de leur sensibilité; soit on définit l'espace comme résultat d'une synthèse opérée par un sujet désincarné qui parcourt l'espace qu'il constitue à son gré. Dans les deux cas l'espace se réduit à un milieu homogène. Ainsi, et le réalisme, et l'intellectualisme se priventils de la possibilité de comprendre la genèse de l'orientation spatiale.

L'ontologie phénoménologique de Merleau-Ponty, en décrivant l'enracinement de notre corps-pratique dans le monde et en déployant les implications de "la foi perceptive», 99 revient sur les abstractions qu'engendre le divorce entre le sujet défini comme un être désincarné sans spatialité et l'objet placé dans un espace géométrico-mécanique. Le sol de l'expérience ne bouge pas, même si la physique démontre avec succès le mouvement de la terre et la relativité de l'espace. Car la dynamique des objets dans l'espace mécanique, expliquée selon la causalité, n'est que la conception abstraite d'une dynamique plus originaire: la dynamique qui naît de l'incompossibilité des choses coexistant dans une simultanéité «sauvage». N'existant que pour un sujet ancré dans le monde, qu'à partir d'un point zéro, les choses n'apparaissent dans leur simultanéité qu'en revendiquant une présence qui n'est jamais pleine, mais qui peut être toujours étoffée. C'est cet apparaître

99. Merleau-Ponty: Le visible et l'invisible, op.cit., 17-74. 
dans l'éloignement qui crée son lieu d'apparition et l'orientation spatiale.

Si la chose ne se spatialise qu'en s'imposant au premier-plan et en repoussant les autres au fond, le monde est déjà toujours traversé par l'imaginaire autant que par le réel. L'espace s'avère être un milieu riche et polymorphe qui se prête autant à l'abstraction imaginative opérée par la physique qu'aux multiples formes de représentation d'autres spatialités: le dessin d'enfant aussi bien que l'art primitif, la perspective picturale classique aussi bien que ses diverses variations, combinaisons et déformations, l'espace poétique aussi bien que l'espace vécu dans l'hallucination. La phénoménologie rappelle ici que «toutes les connaissances s'appuient [...] finalement sur notre communication avec le monde comme premier établissement de la rationalité». ${ }^{100} \mathrm{~L}$ 'espace classique, euclidien ou mécanique, perd alors son privilège, sans perdre sa valeur. Il coexiste avec d'autres conceptions possibles, même si cette coexistence n'est pas toujours sans querelle. Ainsi l'Annonciation des frères Lorenzetti n'est pas l'exemple d'une représentation perspective qui ne serait pas encore parfaite, mais la réalisation d'une forme symbolique où plusieurs modes d'expression spatiale se côtoient. La peinture rend dès lors la vision et l'espace visible et possède une signification ontologique. ${ }^{101} \mathrm{~L}$ 'enfant qui dessine un soleil, à côté d'une maison, aussi grande qu'elle, exprime la perception juste d'une «ultrachose». ${ }^{102}$ Le paradoxe, décrit par Bachelard, d'une armoire dont l'intimité semble être plus profonde que la chambre dans laquelle l'armoire se trouve, ne relève pas de la pure fiction poétique, mais de la réalité de notre expérience. ${ }^{103}$ La poétique de l'espace et l'espace physique ne s'excluent pas l'un l'autre. L'espace poétique est aussi réel et aussi imaginaire que l'espace euclidien. En un mot, l'éventail de Mallarmé, n'est pas une simple métaphore, mais peut littéralement faire «frissonner» l'espace.

100. Merleau-Ponty: Phénoménologie, op.cit., avant-propos.

101. Merleau-Ponty: L'œil et l'esprit, op.cit. 61.

102. Cf. Henri Wallon: Les origines de la pensée chez l'enfant, Paris 1989, 107.

103. Gaston Bachelard: La poétique de l'espace, Paris 1957, 82-85. 


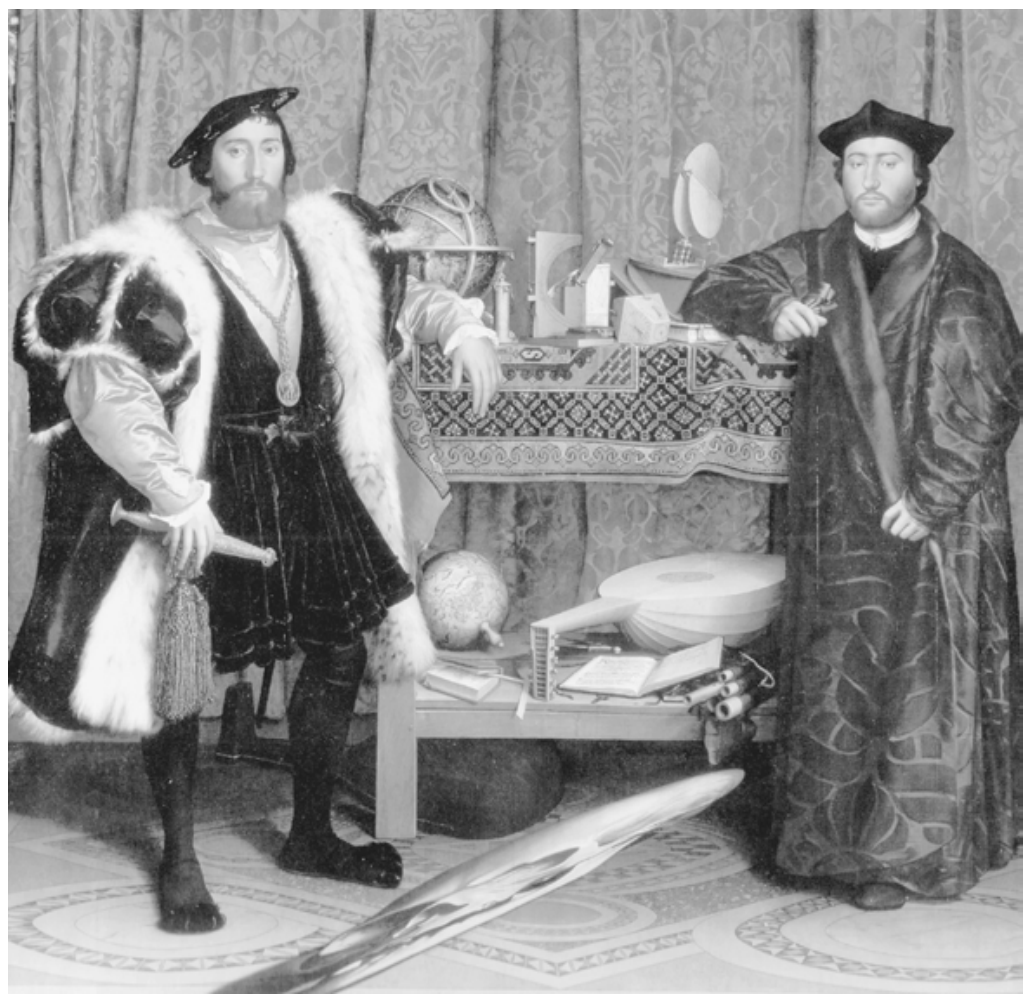

Hans Holbein d.J.: Die Gesandten. 1533. Öl auf Eichenholz, 207 x 209,5 cm. London, National Gallery, ex: Oskar Bätschmann, Pascal Griener: Hans Holbein, Paris 1997, 183. 